Case Report

\title{
A Case of Secondary Leukemia Subsequent to Myelodysplastic Syndromes Successfully Treated with Azacitidine
}

\author{
Takahiro Kumode, Ayano Fukui, Go Eguchi, Terufumi Yamaguchi, and Yasuhiro Maeda
}

National Hospital Organization, Osaka Minami Medical Center, 2-1 Kidohigashi, Kawachinagano, Osaka 586-8521, Japan

Correspondence should be addressed to Yasuhiro Maeda; ymaeda@ommc-hp.jp

Received 7 February 2014; Accepted 16 March 2014; Published 31 March 2014

Academic Editor: Thomas R. Chauncey

Copyright (C) 2014 Takahiro Kumode et al. This is an open access article distributed under the Creative Commons Attribution License, which permits unrestricted use, distribution, and reproduction in any medium, provided the original work is properly cited.

\begin{abstract}
Elderly patients with secondary acute myeloid leukemia (AML) following myelodysplastic syndrome (MDS) are often medically unfit for or resistant to chemotherapy, and their prognosis is dismal. In the present paper, we reported a case of secondary leukemia following MDS in an 80-year-old male patient who was deemed unfit for chemotherapy owing to his old age and poor physical condition. Despite a high tumor burden, treatment with AZA exerted a remarkable response, leading to an immediate cytoreduction in our case. Our results suggest that AZA can be an attractive therapeutic option for elderly MDS or AML patients, offering adequate efficacy and high tolerability.
\end{abstract}

\section{Case and Introduction}

An 80-year-old Japanese man who was originally diagnosed with myelodysplastic syndromes (MDS) and refractory anemia with excess of blasts- 2 in November 2011 was admitted to our hospital in December 2011 owing to the development of acute myeloid leukemia (AML). Prior to admission, the patient had refused therapy for MDS. His medical history included hypertension, chronic heart failure, chronic atrial fibrillation, and dementia. Laboratory findings at admission revealed a white blood cell (WBC) count of $3.3 \times 10^{9} / \mathrm{L}$ with $85 \%$ blast cells, a hemoglobin $(\mathrm{Hb})$ level of $7.5 \mathrm{~g} / \mathrm{dL}$, and a platelet count of $65 \times 10^{9} / \mathrm{L}$. Wilms' tumor gene (wt1) mRNA level in the peripheral blood was 12,0000 copies/ $\mu \mathrm{g}$ of RNA. Bone marrow aspiration showed 56.4\% myoblasts, and Gband karyotype analysis revealed 46,XY,del(20)(q11.2q13.1) [13/20]. Surface marker analysis demonstrated that the blast cells were cluster of differentiations (CD)2-, CD7+, CD19-, CD13+, CD14-, CD15-, CD33-, CD41-, CD61-, CD64-, CD65-, CD11b-, CD34+, CD56-, CD117+, and human leukocyte antigen-death receptor+. The patient was deemed medically unfit for chemotherapy because of his old age and poor physical condition. Therefore, he was started on azacitidine (AZA) at $100 \mathrm{mg}$ /day intravenously for 5 consecutive days every 4 weeks. As shown in Figure 1, after
2 cycles of AZA therapy, he achieved complete remission (CR) according to the Cheson criteria [1] despite of residual $\operatorname{del}(20)(q 11.213 .1)$ clone.

The patient was able to maintain CR and a normal peripheral blood wtl mRNA level without any AML therapy for almost a year. However, his disease relapsed with a high tumor burden, and he was readmitted to our hospital in November 2012 (Figure 2). Laboratory analysis of peripheral blood revealed a WBC count of $104.5 \times 10^{9} / \mathrm{L}$ with $95 \%$ blast cells, an $\mathrm{Hb}$ level of $7.9 \mathrm{~g} / \mathrm{dL}$, and a platelet count of $71 \times$ $10^{9} / \mathrm{L}$. AZA therapy was once again initiated, resulting in a rapid reduction of peripheral blood blast cell percentage to $<5 \%$ immediately after therapy started. After 2 cycles of AZA therapy, the bone marrow demonstrated persisting multilineage dysplasia with a reduction of blasts to $<5 \%$, indicating CR despite of residual del(20)(q11.213.1) clone. Although the patient continued to receive AZA therapy, he succumbed to disease progression in July 2013, which was 20 months after his original diagnosis of AML (Figure 2).

\section{Discussion and Conclusion}

Elderly patients with secondary AML following MDS are often medically unfit for or resistant to chemotherapy, and 


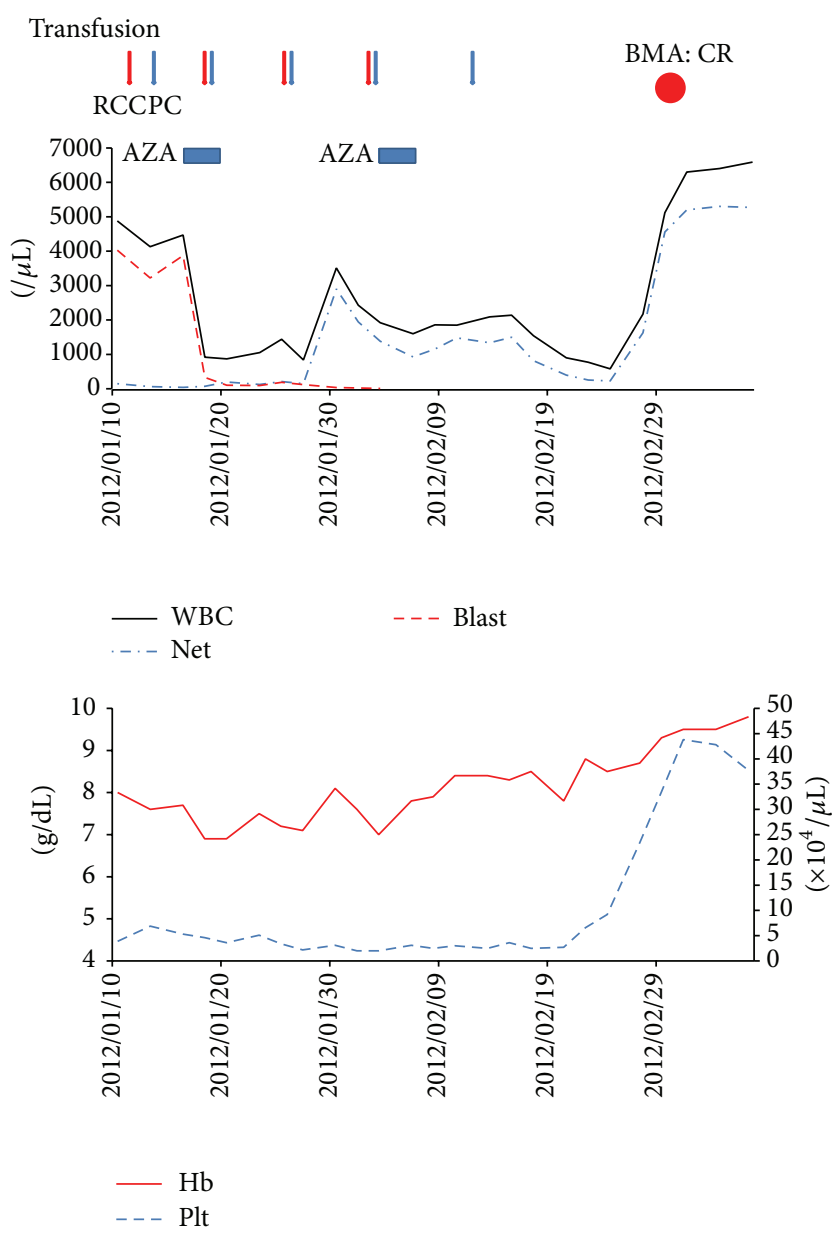

Figure 1: Clinical course after disease onset. RCC: red cell concentrates, PC: platelet concentrates, Aza: azacitidine, BMA: bone marrow aspiration, CR: complete remission, WBC: white blood cells, Net: neutrophils, $\mathrm{Hb}$ : hemoglobin, and Plt: platelets.

their prognosis is dismal. The Cancer and Leukemia Group B and AZA-001 studies have confirmed the efficacy of AZA in high-risk MDS patients whose bone marrow blasts were $\geq 20 \%$ but $<30 \%$, which currently meets the criteria for a diagnosis of AML according to the World Health Organization Guidelines [2,3]. Subsequently, several other studies have suggested that AML patients could benefit from AZA therapy. In a study involving $82 \mathrm{AML}$ patients, including 25 with secondary AML subsequent to MDS, Maurillo et al. reported that AZA was a potentially effective therapy for elderly patients who had previously untreated AML with a WBC count of $<10 \times 10^{9} / \mathrm{L}$ [4]. Al-Ali et al. studied 40 AML patients, including 20 newly diagnosed and 20 relapsed or refractory cases, who were deemed medically unfit for or resistant to chemotherapy. Among these patients, 16 had AML secondary to MDS. All enrolled patients were treated with AZA. Those with newly diagnosed AML experienced a median survival time of 7.7 months and an estimated 1-year survival of $39 \%$, with a median follow-up period of 13 months. These reported results were superior to the published outcomes of standard nonintensive treatments. In addition, the median

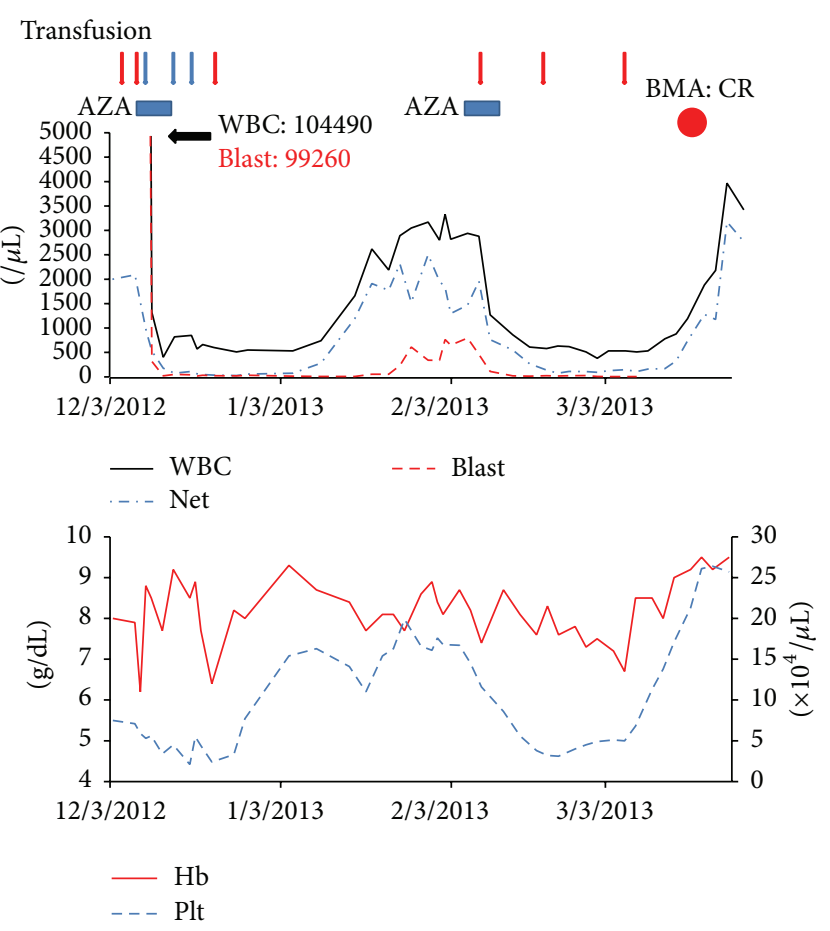

FIGURE 2: Clinical course after disease relapse.

survival time of newly diagnosed AML patients who achieved stable disease was statistically similar to that of patients in CR, partial remission, or hematological improvement [5]. More recently, in a single-institutional study enrolling 227 consecutive older AML patients, including 74 with secondary AML, van der Helm et al. retrospectively compared the efficacy and tolerability of AZA, intensive chemotherapy, and best supportive care. They reported that in comparison to intensive chemotherapy, AZA resulted in a comparable overall survival but higher tolerability [6]. These observations suggest that, unlike intensive chemotherapy, achieving CR with AZA might not be necessary for an improved survival in elderly AML patients. Currently, no biological prognostic factors have been established to predict AZA's efficacy in MDS or AML patients. Itzykson et al. investigated the effect of the ten-eleven translocation 2(TET2) gene mutations on AZA's efficacy in $86 \mathrm{MDS}$ or low blast count AML patients and reported that the presence of a TET2 mutation predicted a higher response rate to AZA than the presence of wildtype TET2 did. However, response duration and survival were comparable between mutated and wild-type groups [7]. On the other hand, Voso et al. showed that TET2 mutations did not have impact on the response to AZA and there were no differences of duration of response and survival between mutated and wild-type groups [8]. More recently, several researches about predictive markers in MDS after treatment of hypomethylating agents were reported [9-12]. According to some reports, TP53 mutations did not predict response to AZA and their prognosis was identically dismal [9-11]. Although the patients group was small, patients with del $20 \mathrm{q}$ might have higher response to AZA [9]. Actually, our present 
case had del20q as well and was seen as remarkable response to AZA. Ahn et al. reported that SRSF2, spliceosomal gene, mutation might be regarded as a risk factor of leukemic transformation compared with other spliceosomal gene mutations [12]. It has been suggested that the efficacy of AZA in AML patients with high tumor burden is limited and that cytoreductive chemotherapy is necessary for these patients. However, in the present case with a high tumor burden, AZA exerted a remarkable response, leading to an immediate cytoreduction similar to what is observed with other intense chemotherapy regimens. Therefore, our results suggest that there are good responders to AZA among both MDS and AML patients. If these good responders could be identified via useful biological prognostic factors prior to the beginning of therapy, treatment with AZA could be encouraged. In conclusion, AZA can be an attractive therapeutic option for elderly MDS or AML patients who are deemed medically unfit for or resistant to chemotherapy.

\section{Conflict of Interests}

The authors declare that there is no conflict of interests regarding the publication of this paper.

\section{References}

[1] B. D. Cheson, J. M. Bennett, K. J. Kopecky et al., "Revised Recommendations of the International Working Group for diagnosis, standardization of response criteria, treatment outcomes, and reporting standards for therapeutic trials in acute myeloid leukemia," Journal of Clinical Oncology, vol. 21, no. 24, pp. 4642-4649, 2003.

[2] P. Fenaux, G. J. Mufti, E. Hellström-Lindberg et al., "Azacitidine prolongs overall survival compared with conventional care regimens in elderly patients with low bone marrow blast count acute myeloid leukemia," Journal of Clinical Oncology, vol. 28, no. 4, pp. 562-569, 2010.

[3] L. R. Silverman, D. R. McKenzie, B. L. Peterson et al., "Further analysis of trials with azacitidine in patients with myelodysplastic syndrome: studies 8421, 8921, and 9221 by the Cancer and Leukemia Group B," Journal of Clinical Oncology, vol. 24, no. 24, pp. 3895-3903, 2006.

[4] L. Maurillo, A. Venditti, A. Spagnoli et al., "Azacitidine for the treatment of patients with acute myeloid leukemia: report of 82 patients enrolled in an Italian compassionate program," Cancer, vol. 118, no. 4, pp. 1014-1022, 2012.

[5] H. K. Al-Ali, N. Jaekel, C. Junghanss et al., "Azacitidine in patients with acute myeloid leukemia medically unfit for or resistant to chemotherapy: a multicenter phase I/II study," Leukemia and Lymphoma, vol. 53, no. 1, pp. 110-117, 2012.

[6] L. H. van der Helm, E. R. Scheepers, N. J. Veeger et al., "Azacitidine might be beneficial in a subgroup of older AML patients compared to intensive chemotherapy: a single centre retrospective study of 227 consecutive patients," Journal of Hematology and Oncology, vol. 6, article 29, 2013.

[7] R. Itzykson, O. Kosmider, T. Cluzeau et al., "Impact of TET2 mutations on response rate to azacitidine in myelodysplastic syndromes and low blast count acute myeloid leukemias," Leukemia, vol. 25, no. 7, pp. 1147-1152, 2011.

[8] M. T. Voso, E. Fabiani, A. Piciocchi et al., "Role of BCL2L10 methylation and TET2 mutations in higher risk myelodysplastic syndromes treated with 5-Azacytidine," Leukemia, vol. 25, no. 12, pp. 1910-1913, 2011.

[9] A. Kuendgen, C. Muller-Thomas, P. Urbaniak et al., "Possible biomarkers to predict response in patients with myelodysplastic syndromes (MDS) or acute myeloid leukemia (AML) treated with 5-azacitidine," Blood, vol. 122, no. 21, abstract 2757, 2013.

[10] A. Kulasekararaj, A. Mohamedali, A. Smith et al., "Comprehensive mutational screening of 5-azacitidine treated myelodysplastic syndrome (MDS) patients fails to identify a specific mutational profile predicting response to therapy," Blood, vol. 122, no. 21, abstract 2792, 2013.

[11] C. Muller-Thomas, M. Rudelius, I. Rondak et al., "Response to azacitidine is independent of TP53 mutations in higherrisk myelodysplastic syndromes (MDS) and secondary acute myeloid leukemia (sAML)," Blood, vol. 122, no. 21, abstract 2797, 2013.

[12] J. S. Ahn, H. R. Kim, H. J. Kim et al., "Clinical significance of SF3B1, U2AF1 and SRSF2 spliceosomal gene mutations for the treatment of hypomethylating agents in myelodysplastic syndrome," Blood, vol. 122, no. 21, abstract 2802, 2013. 


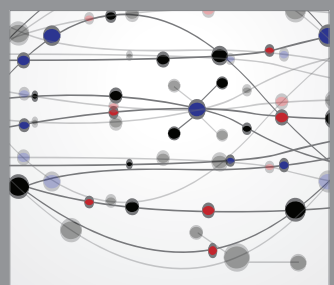

The Scientific World Journal
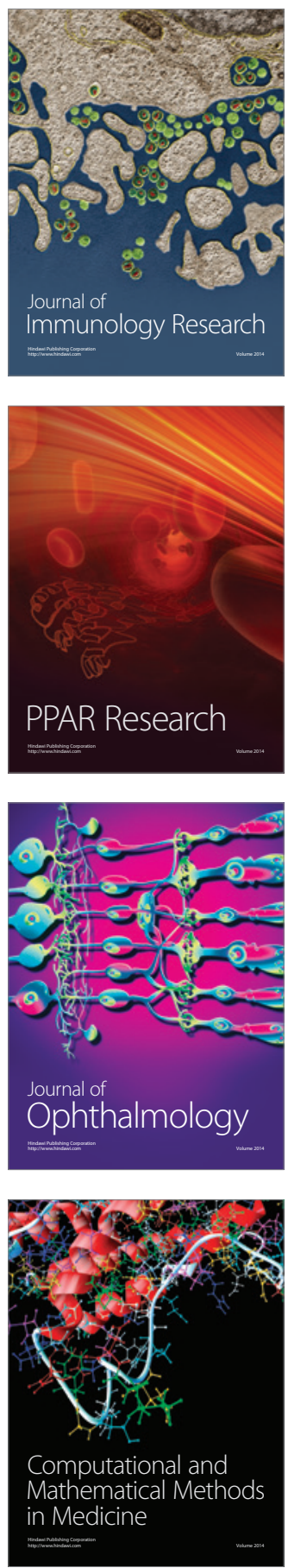

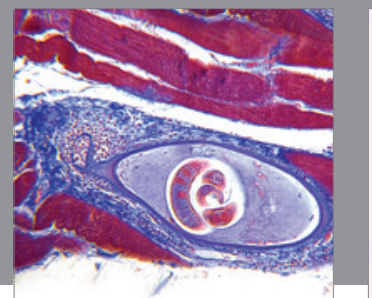

Gastroenterology

Research and Practice
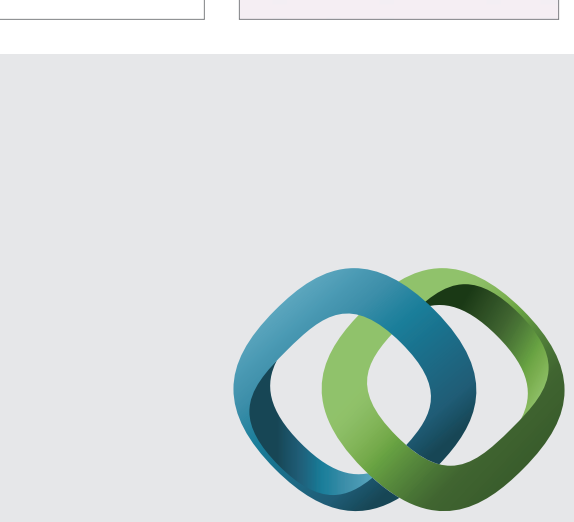

\section{Hindawi}

Submit your manuscripts at

http://www.hindawi.com
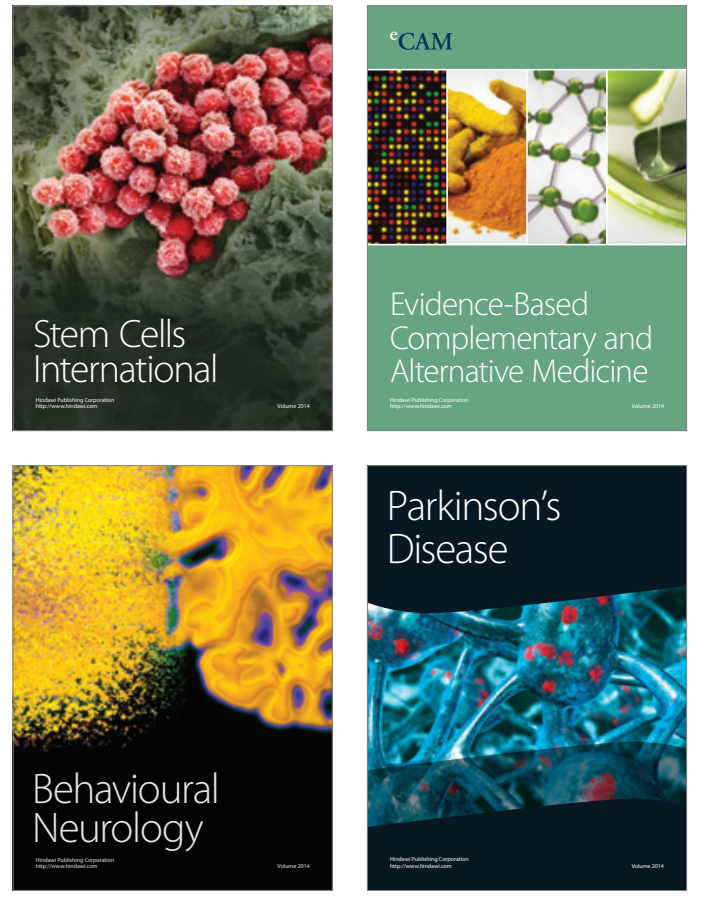
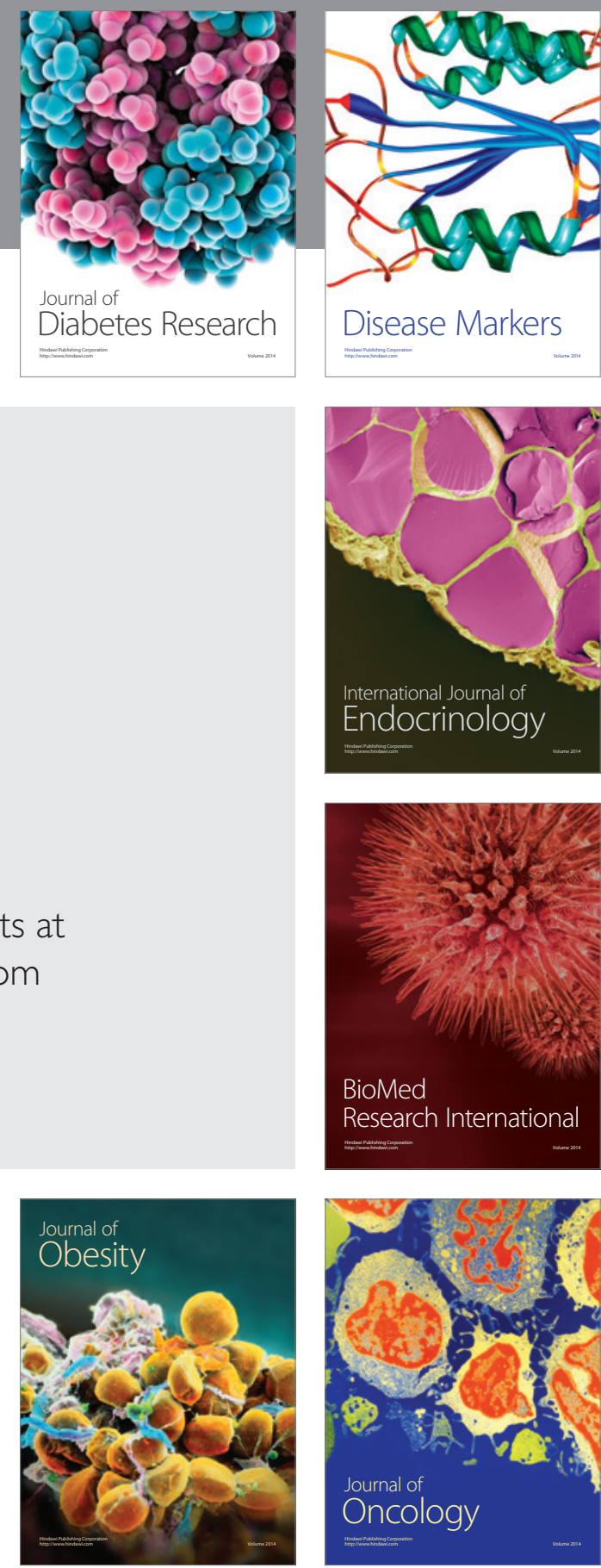

Disease Markers
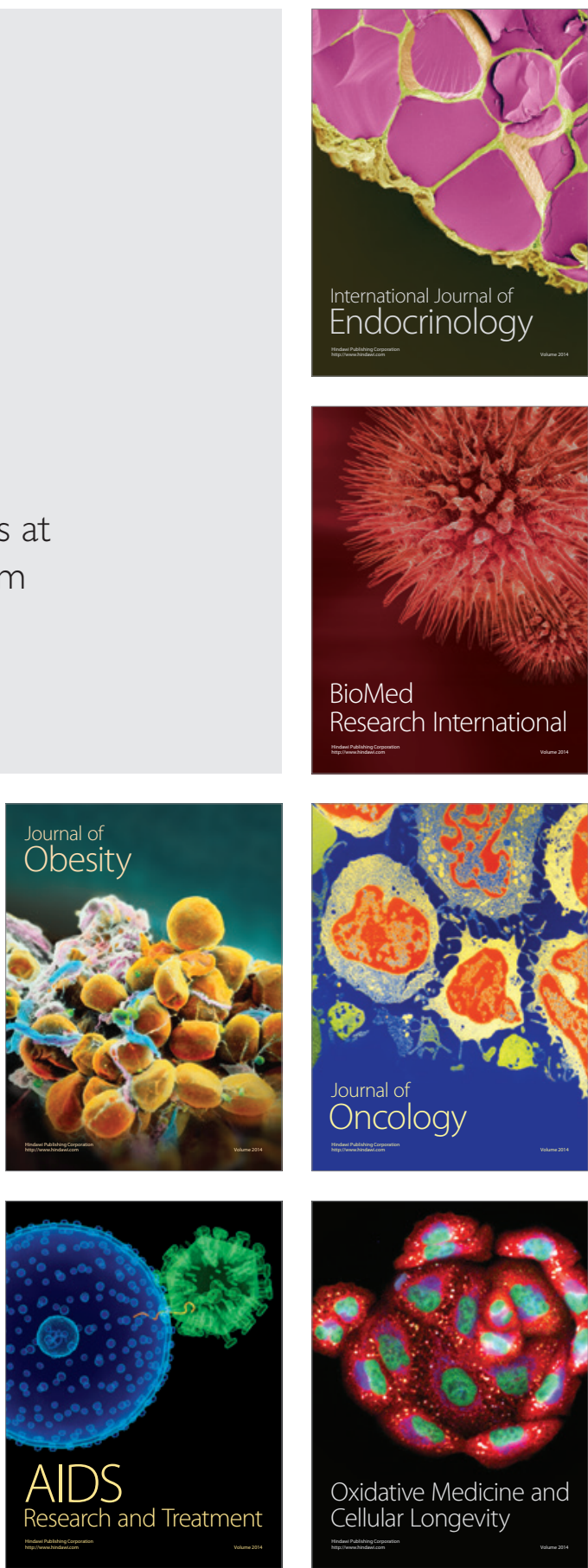